\title{
Utilisation des plantes médicinales dans le traitement des morsures de serpent dans le cercle de Kolokani au Mali.
}

Use of the medicinal plants in the treatment of the bites of snake in the circle of Kolokani in Mali.

Bah $S^{1,2^{*}}$, Maiga $S^{1}$, Dabo $A^{1}$, Diarra $S^{2}$, Sacko $M^{1}$, Sanogo $R^{1,3}$, Diallo $D^{1,3}$.

${ }^{1}$ Faculté de Médecine, de Pharmacie et d'Odontostomatologie, Bamako, Mali.

2Pharmacie Hospitalière du Centre Hospitalier Universitaire du Point G, Bamako

3Institut National de Recherche en Santé Publique, Département de Médecine Traditionnelle, Bamako, Mali

Remerciements : Nous remercions les tradipraticiens, les autorités administratives, communautaires et sanitaires de du cercle de Kolokani pour leur participation et le Bureau OMS de Bamako pour le financement de l' étude.

\section{Résumé}

Le succès du traitement des morsures de serpent en milieu rural passe par l'implication des tradipraticiens de santé.

Le but de cette étude effectué auprès des tradipraticiens de santé de Kolokani (Mali) était de répertorier les différentes plantes utilisées dans la prise en charge des morsures de serpents.

Au total nous avons interviewé 21 tradipraticiens de santé (TPS). La majorité des TPS était des hommes (17/21) soit $80,95 \%$. Plus de $71 \%(15 / 21)$ des enquêtés n'étaient pas alphabétisés. $69,91 \%$ (13/21) des TPS exerçaient leur profession depuis plus de 20 ans. La quasi-totalité des TPS enquêtés soit 95\% (20/21) pouvait nous citer au moins un problème de santé dans la communauté.

Pour la prise en charge des morsures de serpent, au total 16 plantes appartenant à 11 familles botaniques ont été identifiées. Les trois premières plantes les plus citées ont été: Securidaca longipedunculata Fres (Polygalaceae) Gardenia ternifolia.....(Rubiaceae) et Tamarindus indica L. (Ceasalpiniaceae). Les feuilles étaient utilisées majoritairement et le plus souvent sous forme de décocté pour boisson et poudre pour massage. Mots clefs : Enquête ethnobotanique, Tradipraticien de santé, morsures de serpent, Plante médicinales.

\section{Introduction}

La morsure de serpent est la conséquence de la rencontre d'un homme et d'un serpent. Les activités économiques et les occupations du premier, l'écologie et les comportements du second permettent d'expliquer cette rencontre [1].

L'envenimation est l'ensemble des symptômes liés à l'inoculation à l'homme du venin à la suite d'une morsure d'animaux venimeux. L'une des envenimations les plus dangereuses et les plus fréquentes sont celles causées par les serpents, susceptibles d'entraîner la mort par des troubles neurologiques et/ou hématologiques.

Les Elapidés et les Vipéridés sont les deux familles de serpents venimeux les plus fréquentes dans la sous région ouest africaine et sont responsables d'envenimation grave [2].

L'envenimation se voit dans presque tous les pays du monde, mais elle est plus fréquente dans les pays tropicaux. L'activité professionnelle et l'âge constituent des facteurs de risque aux envenimations. C'est ainsi que les agriculteurs, les éleveurs de même que les enfants sont les plus touchés.
Le taux d'incidence et la gravité des envenimations par morsure de serpent sont difficiles à estimer en raison de l'absence de statistiques fiables. Selon les statistiques récentes, il y'a chaque année dans le monde environ 5 millions de personnes victimes de morsure de serpent, avec 2,5 millions de cas d'envenimations, parmi lesquels 125000 en meurent [3]. L'incidence et la mortalité par morsure de serpent sont particulièrement élevées en Afrique, en Asie, en Amérique Latine et en Papoua Nouvelle-Guinée. En Inde on enregistre plus de 50000 décès par morsure de serpent par an. Dans les régions Est de Népal pour une population de 100000 personnes, 162 meurent par morsure de serpent. Pour une population de 750 millions de personnes, 1 million de morsures de serpent surviennent chaque année en Afrique. Au nombre des 500000 envenimations qui en découlent, 20000 décès sont enregistrés dont la moitié seulement est connue des services de santé [4]. En effet on estime à moins de $40 \%$ la proportion de victime qui à la suite d'une morsure de serpent vient consulter dans un centre de santé [4]. Diverses études ponctuelles ont permis d'évaluer la létalité et la fréquence des complications. Une évolution fatale se rencontre dans $5 \%$ des morsures de serpents et que 25 à $40 \%$ des morsures de serpents sont asymptomatiques ou bénignes [5].

Au Mali, le service des urgences de l'Hôpital Gabriel Touré a enregistré une prévalence de 721 pour 100000 consultations par an avec une létalité annuelle de $7 \%$ [6]. Les accidents d'envenimations posent d'énormes problèmes de prise en charge du fait de la mauvaise qualité des soins et de l'éloignement des centres de santé rendant une hospitalisation rapide très difficile.

Le traitement moderne des morsures de serpent et/ou d'envenimations repose sur l'immunothérapie qui n'est toujours pas disponible à cause de :

- L'insuffisance de sérum antivenimeux.

- La méconnaissance des besoins en sérum antivenimeux

- La mauvaise utilisation des sérums antivenimeux

- L'insuffisance de formation du personnel de santé sur les sérums antivenimeux

- Le prix élevé du produit pouvant représenter parfois plusieurs mois de revenu d'un ménage de paysan [7]. Au Mali, malgré leur fréquence et leur gravité, peu d'études ont été orientées sur les accidents d'envenimation par morsure de serpent.

Les manifestations cliniques, l'épidémiologie et les conditions de survenues de ces accidents sont mal connues. L'absence de schéma thérapeutique codifié et 
la forte croyance traditionnelle occasionnent la survenue de complications systémiques et locales [8].

II existe de nombreuses séquelles dues aux nécroses locales conduisant souvent à des amputations. En milieu rural le rôle des tradipraticiens de santé de santé surtout la connaissance des plantes médicinale est bien connue. L'intégration de ces tradipraticiens de santé dans le système de soin de santé primaire serait une alternative dans la prise en charge des morsures de serpent au vu du coût des soins modernes, mais aussi les diversités socio-économiques et socio-culturelles.

C'est dans le but de répertorier les différentes plantes utilisées pour la prise en charge des morsures de serpent dans le cercle de Kolokani que nous avons entrepris cette étude dont l'objectif était d' Etudier les différentes plantes utilisées pour la prise en charge des morsures de serpent dans le cercle de Kolokani.

\section{Méthodologie \\ Lieu d'étude}

L'étude s'est déroulée dans cinq CSCOM et le CSRef du cercle de Kolokani (Figure 1).

Période d'étude et Type d'étude

L'étude s'est déroulée de février à mars 2009.

Nous avons réalisé une étude ethnobotanique qui nous a permis de déterminer les pratiques des tradipraticiens de santé en matière de prise en charge des morsures et envenimation par les serpents, d'identifier les plantes utilisées pour le traitement des morsures et envenimation et de déterminer les parties des plantes ainsi que les modes de préparation des remèdes.

Population d'étude

Vingt un (21) tradipraticiens de santé ont été retenus pour l'enquête ethnobotanique. Les 21 tradipraticiens ont été tirés au sort parmi les tradipraticiens de Tiencoulou et de la ville de Kolokani et de façon proportionnelle au nombre de tradipraticiens de la localité. Le choix des tradipraticiens de Kolokani se justifie pour des raisons de commodité. En effet nous avons choisi les tradipraticiens organisés en association et collaborant avec la médecine conventionnelle. Le choix de Tiencoulou se justifie par le fait que l'étude communautaire s'y est déroulée.

\section{Plan de collecte et d'analyse des données}

Collecte des données auprès des tradipraticiens de santé: l'enquête a été réalisée en utilisant un guide d'entretien présenté.

\section{Définition des variables}

Les variables suivantes ont été définies.

Les plantes: il s'agit des plantes utilisées par les tradipraticiens de santé pour les morsures de serpent. Les plantes ont été identifiées par leurs noms en bambara et botanique avec les familles botaniques.

Les parties des plantes: il s'agit des parties de plantes utilisées. II peut s'agir des feuilles, racines, écorces de tronc, fruits, fleurs, parties aériennes, plantes entières.

Mode de préparation : il s'agit du mode de préparation des plantes à savoir: décoction, infusion, poudre en application, fumigation, etc.

Saisie et analyse des données
Les données ont été recueillies et stockées sur des fiches d'enquête. A la fin de chaque journée, nous avons procédé à une vérification systématique de l'ensemble des fiches remplies pour s'assurer qu'elles ont été bien remplies et corriger éventuellement les erreurs le jour même ou le lendemain. Les données ont été saisies sur Word, Excel et SPSS et analysées sur SPSS 12.0 pour Windows.

\section{Validité}

Pour augmenter la validité et la consistance des données obtenues, nous avons effectué nous mêmes toutes les interviews. Les questionnaires adressés aux tradipraticiens ont été rédigés en français mais l'interview a été réalisée en Bambara. Ceci a pu conduire à des variations. Pour palier à ces insuffisances les questionnaires ont été pré testé auprès des personnes éligibles pour l'étude. Les résultats des pré-tests nous ont permis de corriger les insuffisances notées.

\section{Résultats}

Caractéristiques sociodémographiques des tradipraticiens de santé

Au total nous avons interviewé 21 tradipraticiens de santé dont six (6) à Tiencoulou (village d'étude communautaire) et quinze (15) dans la ville de Kolokani. La majorité des tradipraticiens de santé était des hommes (17/21) soit $80,95 \%$. Plus de $71 \%$ (15/21) des enquêtés n'étaient pas alphabétisés, $23,81 \%$ de nos enquêtés avaient le niveau primaire de scolarisation et seulement $4,76 \%$ (1) avait atteint le niveau secondaire. La majorité $(13 / 21)$ soit $69,91 \%$ des tradipraticiens exerçait la profession depuis plus de 20 ans. La quasitotalité des enquêtés soit 95\% (20/21) pouvait nous citer au moins un problème de santé dans la communauté.

Plantes utilisées contre les morsures de serpent

Plus de la moitié avaient déclaré posséder des remèdes contre les morsures de serpent 80,95\% (17/21). Les remèdes utilisés par les tradipraticiens sont indiqués dans le tableau I. Les tradipraticiens de santé ont cité 16 plantes appartenant à 11 familles botaniques qu'ils utilisent contre les morsures de serpent. Ces plantes sont soit utilisées seules soit en combinaison avec d'autres plantes ou animaux (Tableau $\left.\mathrm{N}^{\circ} \mathrm{I}\right)$. Les plantes ainsi que les parties et les modes de préparation sont mentionnés dans le Tableau $\mathrm{N}^{\circ}$.

Securidaca longipedunculata (Diro en Bambanankan local) a été la plante la plus citée soit $53 \%(9 / 17)$. La deuxième plante la plus utilisée a été Gardenia ternifolia (Bouretié en Bambanankan) $17,65 \%$ (3/17) et la troisième plante était Tamarindus indica (Tomi en Bamabankan) $11,76 \%$ (2/17). II faut noter que le Tomi était toujours utilisé avec d'autres plantes. Les feuilles, les racines et les écorces étaient les parties des plantes les plus utilisées. Les modes de préparation les plus utilisés étaient la décoction et la poudre pour application locale. Nous avons enregistré trois plantes qui n'ont pas pu être identifiées par leurs noms botaniques.

\section{Discussion}


Malgré tous les progrès réalisés en médecine moderne surtout en matière de prévention, de diagnostic et de traitement, l'utilisation des plantes médicinales reste le premier réflexe pour les soins de santé primaire, pour bon nombre de civilisation aujourd'hui surtout les ruraux. Les morsures de serpent en milieux rurales sont traités avec les extraits des plantes affirmaient certains auteurs [10-15]. Dans notre étude centrée uniquement sur la prise en charge traditionnelle des morsures de serpents, nous avons recensé seize (16) plantes médicinales utilisées dans le traitement des morsures et envenimations ophidiennes. $\mathrm{Ce}$ nombre pourrait s'expliquer par le nombre restreint de tradipraticiens concernés $(n=21)$. En effet l'étude a porté sur 21 tradipraticiens de santé exerçant dans le cercle de Kolokani. Les hommes étaient les plus nombreux soient $80,95 \%$ (17/21), expliquant la dominance des hommes dans la profession, cette remarque a été observée au cours d'une étude similaire au Kenya où sur 212 personnes interrogées les hommes représentaient $58,49 \%$ (124/212) [16]. Ils étaient à majorité non alphabétisés $71,43 \%$ (15/21) contrairement à l'étude Kényane ou plus de la moitié étaient scolarisée $40 \%$ niveau primaire, $10 \%$ niveau supérieur et $4 \%$ alphabétisés à l'age adulte [16]. Cette fréquence élevée des non alphabétisés pourrait être expliquée par la faible scolarisation en milieu rural. Parmi les enquêtés $95,24 \%$ $(20 / 21)$ pouvaient nous cité au moins un problème de santé dans la population, donc de véritable généraliste en matière des soins de santé primaire. Ils affirmaient à $80,95 \%$ (17/21) avoir des remèdes contre les morsures et envenimations ophidiennes. Parmi les plantes recensées quatre (4) étaient plus utilisées que les autres à savoir Diro ou Securidata longipedunculata en feuilles, racine et écorce ; le Bouretié ou Gardenia ternifolia en racine et rameau sec; le Tomi ou Tamarindus indica en fruit et le Demassoun en gui.

L'usage de l'Allium $s p$, en Amérique du sud comme antivenin a été reporté par une étude réalisée en Amérique du sud [17]. Allium cepa contient du sulfur, huile volatile [18].

L'utilisation fréquente des feuilles et des racines dans les préparations comme antivenin a été rapportée $[19,20]$. Les plantes étaient préparées soit en poudre le plus fréquent ou en décoction; et utilisées généralement en boisson et/ou en massage.

\section{Références}

1. Chippaux JP 2001 Serpents d'Afrique Occidentale et Centrale. Edition de l'IRD, Paris, P292.

2. Gentilini M 1993.Animaux Venimeux, Flammarion Médecine Science, cinquième edition, Paris, P782 :715-721.

3. N Egenl, J Med vol 347 n 5 .
4. Chippaux JP 2002 Venin de serpent et envenimation. Edition de l'IRD, Paris ,288P.

5. Chippaux JP, Rage Andrieux V, Le Mener, Delore V, Charaondiere M, Sagot P, 2002, Epidemiology of snake envenometion in northern Cameroon. Bull soc pathol, Exot 95(3) 184-187.

6. Drame BSI 2002, Accidents d'envenimations par morsure de serpent au service des urgences chirurgicales de l'hôpital Gabriel Touré. Thèse de médecine, FMPOS, Bamako N 121 p82.

7. Mion G, Olive F, 1998 Envenimation par les Viperidae en Afrique. Réanimation en médecine tropicale 349-365.

8. Enda Tiers Monde 1986, les Envenimations dans l'encyclopédie d'Afrique. Larousse Afrique; 4Belgique, 1111-1115.

9. SIS Kolokani, système d'information sanitaire du CSREF de Kolokani, 2009.

10. Mebs D: Notes on the traditional use of plants to treat snake bite in northern Papua New Guinea. Toxicon 2000, 38(2) :299-302.

11. Otero R, Fonnegra $R$, Jimenez $S L$, Nunez V, Alzate SP, Garcia ME, Saldarriga M, Del Valle G, Osorio RG, Diaz A, Valderrama R, Duque A, Velez HN : Snakbites and ethnobotany in the north-west region of Colombia : Part I :traditional use of plants. Journal of ethnopharmacology 2000, 71(3):493-504.

12. Houghton PJ, Osibogun IM: Flowering plants used against snake-bite. Journal of Ethnopharmacology 1993, 39(1) :1-29.

13. Asuzu IU, Harvey AL: The antisnake venom activities of Parkia biglobosa (Mimosaceae) stem bark extract. Toxicon 2003, 42(7) :763-8.

14. Yang LC, Wang F, Liu M: A study of an endothelin antagonist from a Chinese anti-snake venom medicinal herb. Journal of Cardiovascular pharmacology 1998,31:S249-50.

15. Mors WB: Plants against snake-bites. Memoirs Institute Oswaldo Cruz 1991, 86(suppl 2) : 193.

16. Bethwell O Owuor. ; Daniel P Kissangau. ; Kenyan medicinal plants used as antivenin: a comparison of plant usage. Journal of ethnobiology and ethnomedicine, 2006, 8 p.

17. Bennett BC, Prance GT: Introduced plants in the indigenous pharmacopoeia of northern South America. Economic Botany 2000,54(1) :90-102.

18. Pardee WD, Onion. In Microsoft Encarta Encyclopedia Microsoft Corporation ; 2002.

19. Kokwaro JO: Medicinal Plants of East Africa. In Nairobi East Africa Education Publishers; 1994.

20. Watt JM, Breyer-Brandwijk MG: The Medicinal and Poisonous plants of Southern and Eastern Africa. Edinburgh \& London. E. \& S. Livingstone Ltd ; 1962. 
Tableau $\mathbf{N}^{\circ}$ I : Plantes utilisées en cas de morsure de serpent par les tradipraticiens de santé de Tiencoulou et de Kolokani (cercle de Kolokani)

N.B : Les noms vernaculaires sont de bambara local de Kolokani.

\begin{tabular}{|c|c|c|c|c|}
\hline Familles & Noms Scientifiques & Nom Bamanan & $\begin{array}{l}\text { Partie utilisée, } \\
\text { Mode de préparation et d'utilisation }\end{array}$ & Fréquence \\
\hline Fabaceae & Acacia albida Delile & Balazan & $\begin{array}{l}\text { Poudre d'écorce à boire et à appliquer sur le } \\
\text { membre mordu avec du beurre de karité }\end{array}$ & 1 \\
\hline Liliaceae & Allium cepa L. & Diaba & $\begin{array}{l}\text { Feuilles et/ou tubercule broyer, à appliquer sur le } \\
\text { membre mordu }\end{array}$ & 1 \\
\hline Vitaceae & Ampelocissus gradii (Baker) Planch. & $\begin{array}{l}\text { Folokofaloka } \\
\text { où Logolangan }\end{array}$ & Décoction des feuilles à boire & 1 \\
\hline Cochlospermaceae. & Cochlospermum tinctorium A. Rich & $\begin{array}{l}\text { Gouloubara } \\
\text { ou Tiribara }\end{array}$ & $\begin{array}{l}\text { Poudre de tubercule à boire et à mélanger avec le } \\
\text { beurre de karité et appliquer sur la partie mordue }\end{array}$ & 1 \\
\hline Rubiaceae & Crossopteryx febrifuga (Afzel) Benth. & Balembo & $\begin{array}{l}\text { Poudre des fruits à appliquer sur la partie mordue } \\
\text { avec le beurre de karité }\end{array}$ & 1 \\
\hline Solanaceae & Datura metel L. & Almoukakaye & Décoction des feuilles à boire & 1 \\
\hline Rubiaceae & $\begin{array}{l}\text { Gardenia ternifolia Schumach. \& } \\
\text { Thonn. }\end{array}$ & Bourétiè & $\begin{array}{l}\text { Poudre de racines et des rameaux sèches à } \\
\text { appliquer sur le membre mordu avec le beurre de } \\
\text { karité }\end{array}$ & 3 \\
\hline Moringaceae & Moringa oleifera Lam. & & Décoction des feuilles à boire & 1 \\
\hline Fabaceae & Piliostigma reticulatum (DC.) Hochst. & Niamatiè & Décoction des feuilles à boire & 1 \\
\hline Euphorbiaceae & Ricinus communis L. & Tomotiqui & Décoction des feuilles à boire & 1 \\
\hline Anacardiaceae & Sclerocarya birrea (A. Rich.) Hochst & N'gouna & $\begin{array}{l}\text { Poudre d'écorce à boire et/ou à appliquer avec de } \\
\text { beurre de karité sur le membre mordu }\end{array}$ & 1 \\
\hline Polygalaceae & Securidaca longipedunculata Fresen. & Diro & $\begin{array}{l}\text { Décoction de feuilles à boire, poudre de feuilles, de } \\
\text { racine et d'écorce à boire avec de lait ou de l'eau } \\
\text { et/ou à appliquer sur le membre mordu }\end{array}$ & 9 \\
\hline \multirow[t]{7}{*}{ Caesalpiniaceae } & Tamarindus indica L. & Tomi & $\begin{array}{l}\text { Jus ou décoction des fruits à boire, associé } \\
\text { généralement à d'autre plante }\end{array}$ & 2 \\
\hline & & Demassoun & $\begin{array}{l}\text { Poudre de gui mélangée de beurre de karité à } \\
\text { appliquer à la partie mordue }\end{array}$ & 2 \\
\hline & & Tiambaly & Poudre et décoction de feuilles à boire & 1 \\
\hline & & Soubabana & Décoction de feuilles à boire & 1 \\
\hline & & Tibo (oiseau) & $\begin{array}{l}\text { Poudre de la jambe mélanger au beurre du karité à } \\
\text { appliquer sur le membre, à boire avec de l'eau ou } \\
\text { avec de soupe }\end{array}$ & 1 \\
\hline & & Satéké (rongeur) & $\begin{array}{l}\text { Poudre de l'animal à appliquer sur le membre ou à } \\
\text { boire avec un liquide }\end{array}$ & 1 \\
\hline & & $\begin{array}{l}\text { Autres poudres non } \\
\text { spécifiées }\end{array}$ & $\begin{array}{l}\text { Poudre pour massage avec du beurre de karité à la } \\
\text { partie mordue }\end{array}$ & 4 \\
\hline
\end{tabular}

